\title{
Energy-Efficient Dynamic Dominating Set Construction in Wireless Sensor Networks
}

\author{
Dahlila P. Dahnil, Yashwant P. Singh, and Chin-Kuan Ho
}

\begin{abstract}
Clustering and topology control are two well-known techniques to improve network lifetime in Wireless Sensor Networks. This paper proposes a hybrid approach combining both techniques to construct a dominating set that aims to minimize energy consumption by abandoning long-distance communication. A transmission power control strategy is proposed to establish connection for all nodes so as to communicate in one hop to their cluster heads and consequently reducing the energy consumption. Topology control along with a clustering technique elects cluster heads to form a dominating set. The proposed hybrid approach constructs a dynamic dominating set based on energy and coverage metrics with low computational complexity and significant reduction in the amount of message overhead required for dominating set construction. Simulation results show that the set of elected cluster heads is a dominating set constructed dynamically on every round having optimal one-hop cluster.
\end{abstract}

Index Terms-Adaptive clustering, dominating set, energy-efficient, topology control, Wireless Sensor Networks.

\section{INTRODUCTION}

Wireless Sensor Networks have evolved in many aspects and they continue to be networks with constrained resources in terms of energy, computing power, memory and communications capabilities. Among these constraints, energy consumption is of a paramount importance for design considerations. This has led to the development of a number of algorithms, techniques and protocols, employed to save energy and thereby extends the lifetime of the network [1]. One important design approach is clustering, a well-known technique to reduce energy consumption in Wireless Sensor Networks. Nodes are divided into groups, where each group agrees on a central node, called the cluster head, which is responsible for gathering the sensors' data of all group members, aggregating it and sending it to base stations (BS) [2]. Another energy-saving approach is to build a reduced topology that will save energy through topology control. Topology control can be implemented using two approaches which are referred to as transmission-power-based algorithms and duty-cycle-based algorithms [3]. The first approach is via power control by reducing the transmission range of all nodes by the same amount or reducing the minimum transmission range for each node. The goal of power control is to change the nodes' transmission range in order to preserve some communication properties such as

Manuscript received October 14, 2012; revised November 15, 2012. This work is financially supported by Yayasan Khazanah, of Khazanah Nasional Berhad, Malaysia.

D. P. Dahnil is with the Multimedia University, Malaysia (e-mail: dahlilaputri@gmail.com). connectivity and coverage [4].The second approach is to turn off certain nodes for low duty cycle. Various clustering and topology control algorithms have been presented in the literature [5]-[8]. Existing research works reported in the literature address clustering technique and topology control separately. There are attempts to combine the clustering technique and topology control for developing improved energy-efficient solutions but still not widely explored [1], [9]. If a clustering technique is exercised jointly with power control, a new energy-efficient method of constructing dominating set may be obtained in Wireless Sensor Networks.

The proposed algorithm is a hybrid approach to construct dominating set by incorporating the clustering technique where nodes are one-hop to their cluster heads by establishing connection of near neighbours via power control. Having member nodes closed-by the cluster heads eliminate long distance communication that not only reduce energy consumption but also suppress interference due to low power transmission. To balance the energy consumption among nodes in the network, the dominating sets are re-elected and changed at every round [10], [11]. The proposed algorithm is fully distributed, without requiring any initiator node to start the network discovery process. The algorithm is simple and require small message overhead with low computational complexity of $\mathrm{O}(n)$.

The remainder of this paper is organized as follows: Section 2 briefly describes the existing research works on clustering and topology control techniques. Section 3 describes details of the proposed algorithm and a proof that the algorithm produces dominating set on every round. Section 4 provides the simulation results to show the correctness of the proposed hybrid algorithm and Section 5 concludes the paper.

\section{RELATED WORKS}

In a cluster-based approach for a Wireless Sensor Networks formation, different hierarchical level is created in which a reduced subset of nodes is selected and given more responsibility such as information aggregation, filtering, routing and message forwarding. This approach greatly simplifies the network topology and the opportunity to save additional energy by assigning useful functions to reduced subset of nodes or cluster heads [6]. The cluster heads require extensive energy for communication with member nodes and to forward data to base station (BS). Their batteries will deplete sooner than that of the member nodes if the role of cluster heads is not rotated among all nodes in the network. Numerous dynamic clustering schemes have been proposed 
in the literature to allow rotation of cluster heads among nodes [12]. A number of research works has concentrated on the challenges of clustering protocol such as the cluster head election process to overcome the high communication overhead. The paper reported in [11] has presented cluster heads election based on energy consideration while multiple criteria optimization technique is used for cluster heads formation in [13]. Low-energy Adaptive Cluster Hierarchy (LEACH) [10] proposed a single hop self-organized algorithm for WSNs. A sensor node elects itself as cluster head with some probability and broadcast its decision. To balance the overall energy consumption across the network the role of the cluster heads is rotated among all the nodes in the network. However, LEACH does not guarantee a good cluster head distribution and requires expected number of cluster heads a priori. HEED overcomes this drawback and produces non-overlapping clusters and manages the scalability issues [14]. The use of sensors with different sensing or communication and computation capabilities leads to having heterogeneous nodes in the network. These nodes are very helpful for performing additional functionalities or given more responsibilities [15]. Nodes having more computing and communication capabilities are given more chances to become cluster heads compared to normal nodes.

A dominating set (DS) of a graph $G=(V, E)$ is a subset of nodes $V$ such that each node $v \in V$ in the graph is either in the subset or adjacent to at least one of the node in that subset [6]. Various approaches have been proposed for constructing dominating set (DS) mainly in Mobile Ad Hoc Networks [7, 16, and 17]. A dominating set is always desirable in order to enable a hierarchical structure for coverage problem in the network where the members of the dominating set provide special services or roles to their neighbours. The dominating set in the graph theory is hierarchical cluster-based structure where the cluster heads constitutes a dominating set. The minimum dominating set finds a dominating set of minimum size which has been proven to be NP-hard problem [18]. The best known approximation is achieved by the greedy algorithm. As long as there are uncovered nodes, the greedy algorithm picks a node which covers the biggest number of uncovered nodes and puts it into the dominating set. [19]. Topology Management by Priority Ordering (TMPO) [16] builds a hierarchical topology combining clustering technique and dominating set in mobile ad hoc networks. TMPO uses clustering to build a minimal dominating set and selects special nodes called doorways and gateways to build a connected dominating set. In TMPO, node decides to become a cluster head if it has the highest priority in its one-hop neighbourhood, or if it has the highest priority in the one-hop neighbourhood of one of its one-hop neighbours. However, TMPO is aimed towards mobile ad-hoc networks than static Wireless Sensor Networks.

Only few research efforts have tried to integrate the construction of dominating set and cluster-based power control in Wireless Sensor Networks [6]. Research works on topology control A3 algorithm [1], proposed topology construction mechanism that finds a sub-optimal connected dominating set (CDS). The algorithm is based on growing-tree technique and selects active nodes which are at the farthest distance from the parent based on the signal strength and remaining energy. This allows fewer nodes to be selected in the CDS tree which in turn leads to an overhead of long distance communication [20].

The proposed hybrid algorithm constructs a dominating set (cluster heads) such that the dominating sets are chosen to eliminate long-distance communication of direct members of dominating sets by implementing power control. The cluster formation overhead generated is smaller than that of A3 algorithm. Each node chooses a closed-by (nearest neighbour) cluster head. For energy minimization, it is always better to have more number of hops to reduce long distance transmission [19]. For dominating set construction, transmission power level controls the establishment of connection to nearest neighbours. Having only near neighbours not only helps reducing energy but also interference since fewer nodes is disturbed by low power transmission [21]. The members of dominating set may be chosen as relay nodes (cluster heads that helps to relay data from other cluster heads) to route messages from direct neighbours over several small (energy-efficient) hops to BS. Since members perform a short range communication, a reduction in energy consumption leads to improved network's lifetime. Only fraction of nodes (i.e. dominating sets) transmits a long range to BS. Previously dominating sets are constructed based on node degree [19]. The proposed hybrid algorithm considers node energy as selection metric for constructing dominating set. A connected dominating set (CDS) can be obtained through gateway nodes. Nodes within range of more than one cluster heads are suitable candidates of gateway nodes. These nodes can be assigned to forward data between cluster heads to form connected cluster heads.

The simulation results show the number of elected cluster heads (dominating set) is controlled by the transmission power level used during the clustering process. It also demonstrates the effectiveness of maximizing numbers of dominating set by minimizing the transmission range of nodes compared to minimizing number of dominating sets by setting higher power level. There is a trade off between the establishment of connection of nearest neighbours via controlling power level and the dominating set construction to form a balanced design.

\section{The Proposed Algorithm}

\section{A. Dominating Set in Wireless Sensor Networks}

A Wireless Sensor Network is often represented by a graph in which vertices correspond to sensor nodes, $v$ and bi-directional edge from one vertex, to another indicates that the node corresponding to the former can send data directly to the node corresponding to the latter. The propagation conditions can be modelled simply by there being a transmission range within which transmission is possible [4]. The transmission range is an important property that establishes connectivity which is an important goal for obtaining the connected graph. The proposed algorithm construct dominating set by changing the transmission range while maintaining the network properties such as connectivity and coverage. Each node belongs to a cluster 
head, which is responsible for forwarding data to ensure full coverage of the network.

\section{B. Dynamic Dominating Set Construction}

The proposed algorithm for constructing dynamic dominating set is totally distributed and self-organized. The protocol starts with Phase 1 of neighbour discovery. Any node can initiate the discovery process and does not require any pre-defined node (e.g. the sink node) to initiate the process as in [1], [20]. The neighbour lists are stored by each node after the discovery process and will be used for cluster head election. The neighbour discovery is not necessarily done on every round as the frequency of node failure does not happen very often [22]. Each node $i$, compute the probability of becoming cluster head candidates based on residual energy. Nodes with higher energy elect themselves with higher priority to become cluster head candidates probabilistically. The algorithm aims to have high probability of cluster heads on each region of the network called a square. A square in this context is defined as the area in 2-dimensional space in which minimum allowable distance to ensure connectivity between nodes to communicate with each other. The proposed algorithm constitutes of 5 Phases: Neighbour Discovery, Candidate Election, Cluster Head Election, Member Associations and Support Election. The algorithm proceeds in rounds that follow the setup phase and steady phase as proposed in LEACH. In any round of data reporting during network operation, each node sends one packet to its cluster head and the cluster heads send aggregated data packet to base station. The description of the algorithm is as follows:

Phase 1: Neighbours Discovery - Each node finds its neighbours and stores this information in its neighbour list. This neighbour list will be used later to elect cluster heads.

Phase 2: Candidate Election - A node calculates its probability $\mathrm{P}\left(\mathrm{CCH}_{i}\right)$ to become cluster head candidate based on its residual energy, $E_{i}$ with respect to the average energy of all nodes in the network, $E_{\text {avg }}$. LEACH protocol defines the optimal number of cluster heads, $k_{\text {opt }}$ to achieve minimum energy dissipation per round. We set a clustering parameter where $k_{\text {initial }}>k_{\text {opt }}$ for the election process to get a non-overlapped cluster heads. The $k_{\text {initial }}$ is set to have value $>k_{o p t}$, for example double of $k_{o p t}$, such that only high-energy nodes will be elected and cover the whole network area. A node becomes cluster head candidate with a probability $\mathrm{P}\left(\mathrm{CCH}_{i}\right)$ and is calculated as follows:

$$
\begin{gathered}
P\left(C C H_{i}\right)=\frac{E_{i}}{E_{a v g}} \\
E_{\text {avg }}=\frac{E_{\text {total }}}{k_{\text {initial }}}
\end{gathered}
$$

Each node generates a random number in the range of $[0,1]$ and if the number is less than the calculated probability $P\left(C C H_{i}\right)$, it elects itself as cluster head candidate The average energy of all nodes is obtained when nodes send their current residual energy to their respective cluster heads which aggregate and send this information to other cluster heads in the network. Based on this information, cluster heads will calculate the average energy of all clusters in the network and forward this information to its members for $\mathrm{P}\left(\mathrm{CCH}_{i}\right)$ calculation.

Phase 3: Cluster Head Election - A cluster head candidate $c \in V$ becomes a cluster head under the following conditions: If its coverage is different and not the subset of the neighbouring cluster head candidates, it will elect itself as cluster heads

Phase 4: Member Associations - A node associates itself to a cluster head based on strongest received signal strength. Member re-association can take place for nodes that have become a member of a cluster if member nodes received higher signal strength from other neighbouring cluster heads even after it has joined a cluster. Nodes receiving more than one cluster head messages send gateway message for $\mathrm{CH}$ to identify and elect gateway nodes. These gateways are members to one of the clusters but they are suitable candidates for gateway nodes, in situation where cluster heads cannot reach other cluster heads in the network.

Phase 5: Support Election - Node neither a cluster head nor a member of cluster elects itself as cluster head candidates after the timeout and proceed with Phase 3 again.

\section{Protocol Implementation}

The algorithm starts with Neighbours Discovery followed by Candidate Election. The value set for $k_{\text {initial }}$ is important as it is the key for electing high energy cluster head candidates. If the values is set high, higher probability of having cluster head candidates covering the whole network coverage and therefore, ensuring the elected cluster heads are among nodes of high energy. Each node then sets its status as normal node, called NMNODE and evaluates the probability of becoming cluster head candidate as described in Phase 2. If a random number generated is less than the calculated probability, a node elects itself as cluster head candidate. It then sets its status as CCNODE and broadcast candidate message, CONTENDMSG, within its closest neighbours depending on the power level setting. The message contains the nodes neighbour list. Normal nodes not elected as cluster head candidate set a timer. The cluster head candidate announces itself as cluster head on two situations: (1) if the node is the only cluster head candidate available within that range, (2) if the cluster head candidates receive other candidates' messages, it evaluates its position based on its coverage, from the neighbour list. If the node has different coverage, it announces itself as cluster head, set its status to CHNODE and sends cluster head message, CHMSG. Otherwise it sets itself as CDNODE and knows it is within other candidate nodes coverage. Neighbouring nodes receiving the cluster head message associate itself as member of a cluster based on strongest received signal strength (closed-by cluster head) and set its status as MMNODE. Nodes that have more than one cluster head within its range inform neighbouring cluster heads by sending GWMSG. The cluster heads may choose gateway nodes for forwarding data. Normal nodes which are neither cluster heads nor member of a cluster, elect itself as cluster head candidates after timer expires and proceed to Phase 3. The algorithm ensures all nodes are covered by closed-by cluster heads so that a 
dominating set is obtained. An example of the proposed algorithm is shown in Fig. 1.

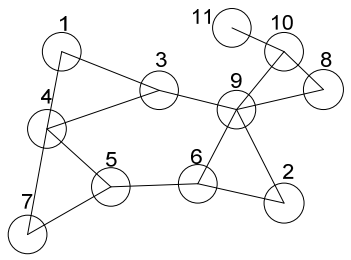

a) Neighbour discovery

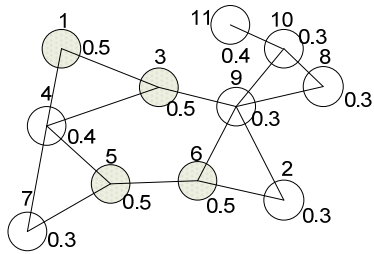

b) Cluster head candidates

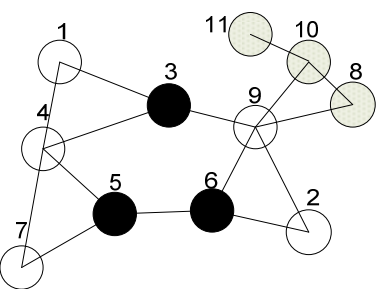

c) Cluster heads elected and re-election of cluster head candidates

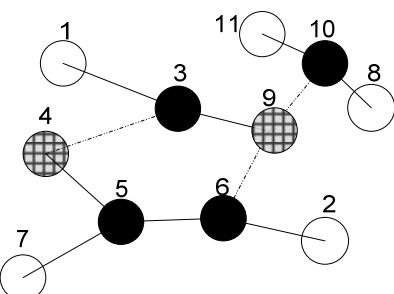

d) Cluster heads and possible gateway nodes

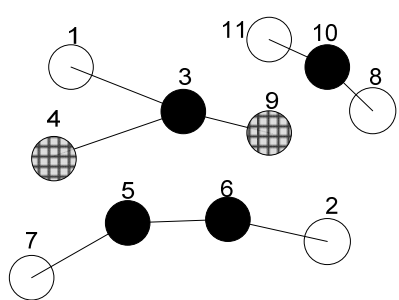

e) Elected cluster heads

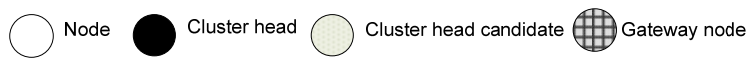

Fig. 1. The dynamic dominating set construction

In Fig. 1a) each node discovers their neighbours and exchanges their neighbour list. Node 1, 3, 5 and 6 are elected as cluster head candidates as they have higher energy as in Fig. 1b). Since node 1's neighbour is subset of 3, it withdraws its candidature and become CDNODE. Node 5 and 6 know they have different neighbour and different coverage therefore, they elect themselves as cluster heads. In Fig. $1 \mathrm{c}$ ) node $1,4,7,9,2$ receive the cluster head messages so they are excluded from the Support Election process. Node 8, 10 and 11 do not receive any message and when the timer expires, they elect themselves as cluster head candidates. Node 8 and 11 withdraw its candidature as they have the same coverage as node 10 . Node 10 elects itself as cluster head as it has more coverage than its neighbours. Fig. 1d) shows the final cluster heads elected. Node 4 and 9 knows they are within more than one cluster heads range therefore, they may become gateway nodes. The pseudo code for the proposed algorithm is given as follows:

Neighbour Discovery;

Calculate $\mathrm{P}\left(\mathrm{CCH}_{i}\right)$;
Generate random number, rnd $\in[0,1]$;

If rnd $<P\left(\mathrm{CCH}_{i}\right)$

Broadcast CONTENDMSG;

Status $=$ CCNODE

End if

Start timer

If Timer expires and Status $=$ NMNODE

Broadcast CONTENDMSG;

Status $=$ CCNODE

End if

While status $=\mathrm{CCNODE}$

If received other CONTENDMSG

If different coverage

Broadcast CHMSG;

Status $=$ CHNODE;

Else

Status $=$ CDNODE;

End if

Else

Broadcast CHMSG;

Status $=$ CHNODE;

End if

End while

If received CHMSG;

Join cluster head with strongest signal strength;

Broadcast GWMSG;

Status $=$ MMNODE;

End if

Through topology control, a one-hop cluster can be obtained by the proposed algorithm. It has been shown through experimental results analyses that clusters in which all nodes can communicate in one hop to the cluster head are optimal [2]. The algorithm can be the basis to construct dominating set to support multi hop communication protocol.

\section{Cluster Head Candidate Election}

We consider cluster formation in a predetermined grid form such as rectangular node placement as a strategy to get high probability of cluster head candidates on a square cell network [23] as shown in Fig. 2.

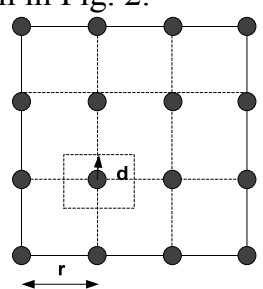

Fig. 2. The placement of nodes in network

For a successful transfer of data to adjacent nodes, minimum communication distance covered, $r$ is taken to be at least twice the sensing range, $d$. The sensing area to be covered by each node is given by $r^{2}$ and the total sensing area covered by n number of sensor nodes is $n r^{2}$. Given an area, $A$ of $m \times m$ dimension, to obtain a full coverage and to have high probability of cluster head candidates on each square the minimum value of $k_{\text {initial }}$ is set as follows:

$$
k_{\text {initial }} r^{2}=m x m
$$


From (3), the transmission range and the network area determine the number of cluster head candidates. If the dimension of the network is set to $100 \mathrm{~m} \times 100 \mathrm{~m}$ with node size 100 and the range is taken as $30 \mathrm{~m}$, the minimum number of cluster head candidates cover the entire network will be approximately 11 . The basis for considering $30 \mathrm{~m}$ range corresponds to average power level of sensor nodes. If this range is increased, less number of cluster head candidates is required and therefore, smaller size of dominating sets is formed after the competition among the cluster head candidates.

\section{E. Message Complexity}

The proposed hybrid protocol constructs dynamic dominating set based on energy and coverage metrics with low computational complexity and significant reduction in the amount of messages required for dominating set construction. During the neighbour discovery each node only has to send out discovery message. Thus the total number of these messages is $\mathrm{O}(n)$ where $n$ is the number of sensor nodes For message exchange in Phase 1 and 2 for cluster head candidates and cluster head election, only partial of nodes send out the message which is less than $n$. In Phase 4, the number of joint messages in the network is strictly less than $n$ since some nodes are the dominating set nodes and thus the total exchange messages in the network is $\mathrm{O}(n)$.

\section{Dynamic Dominating Set Protocol Analysis}

Proof

Lemma1. The set of cluster heads elected by the hybrid algorithm is a dominating set.

Proof:

Assume part of the nodes in the network become cluster head candidates. All cluster head candidates become cluster heads based on coverage of its list of neighbour. If it has different neighbours than its other candidates in the same transmission range, it becomes a cluster head and broadcasts the cluster head message. Neighbour nodes receiving this message are covered and become member of this cluster head based on strongest signal strength. Cluster head candidate becomes normal node only if its coverage is subset of other candidates which ensures its neighbour is covered by a cluster head candidate. Uncovered nodes that are still on normal node status, after timeout expires will become a cluster head candidate. The same election process takes place which means all the uncovered node will be either the cluster head or the member of a cluster.

\section{Simulation Results}

TABLE I: SIMULATION PARAMETERS

\begin{tabular}{|l|l|}
\hline Parameters & Values \\
\hline Network grid & From $(0,0)$ to $(100,100)$ \\
\hline Sink & $(50,175)$ \\
\hline Initial Energy & $0.5 \mathrm{~J}$ \\
\hline Eelec & $50 \mathrm{~nJ} / \mathrm{bit}$ \\
\hline$\epsilon_{\mathrm{fs}}$ & $10 \mathrm{pJ} / \mathrm{bit} / \mathrm{m} 2$ \\
\hline$\epsilon_{\mathrm{mp}}$ & $0.00013 \mathrm{pJ} / \mathrm{bit} / \mathrm{m} 4$ \\
\hline Efusion & $5 \mathrm{~nJ} / \mathrm{bit} / \mathrm{signal}$ \\
\hline Data size & $500 \mathrm{bytes}$ \\
\hline
\end{tabular}

Simulations are carried out to evaluate the proposed algorithm for different network sizes. Simulation considers network size varying from 100 to 400 nodes that are placed uniformly and randomly in the network of dimension $100 \mathrm{~m} \mathrm{x}$ $100 \mathrm{~m}$. The base station is located at $x=50, y=175$. The simulation parameters considered are as described in Table I.

\section{A. Network Lifetime}

We run simulation for different value of $k_{\text {initial }}$ to see the effect on the number of elected cluster head candidates and the coverage on the network. Fig. 3 shows the round first node dies with different value of $k_{\text {initial }}$. For a short range transmission radius, $10 \mathrm{~m}$, the round of the first node death is around 155. This is due to the worst case scenario where there exist single-node cluster heads as this node is in isolation without any neighbours. Once transmission range increases, the load can be distributed among neighbours by rotating the cluster heads and causes improvement on lifetime. Fig. 4 shows the number of cluster head candidates elected (indicated by stars) and its coverage in the network. It can be seen that as the number of $k_{\text {initial }}$ increases from 10 to 30 , the cluster heads candidate coverage improves as higher probability of candidates to be elected and covers more area. This ensures high energy nodes will be elected and distributed on the network area.

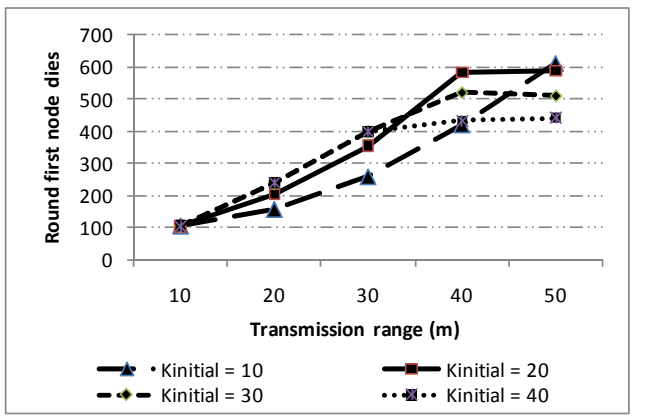

Fig. 3. Round first node dies with different value of $k_{\text {initial }}$

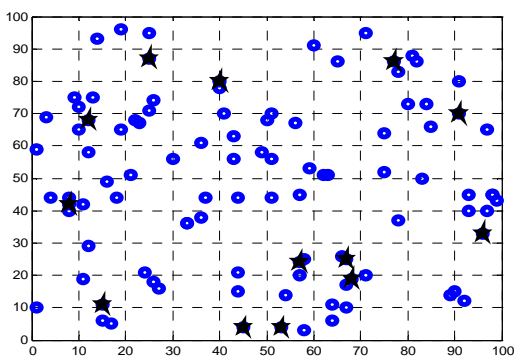

a) $k_{\text {initial }}$ is set to 10

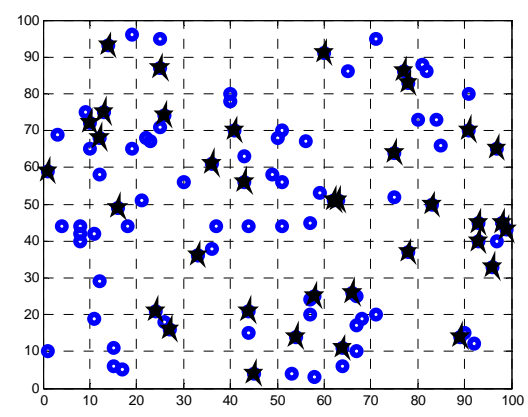

(b) $k_{\text {initial }}$ is set to 30

Fig. 4. The cluster head candidates distribution 


\section{B. One-hop dominating set}

The following simulation results, Fig. 5 and Fig. 6, show the construction of dominating set when the transmission range is set at $10 \mathrm{~m}$ with two different network sizes of 100 and 400 respectively. One-hop dominating set is obtained due to power level setting as it controls the size of neighbours' nodes. The combination of power control and clustering technique shows the ability to create one-hop dominating set or the option to control the dominating set. As indicated by [2] from its experimental results analysis, cluster in which all nodes can communicate in one hop to the cluster heads are optimal. Since energy consumption for communication increases with distance [5], maximizing the number of hops through relay nodes helps to save energy.

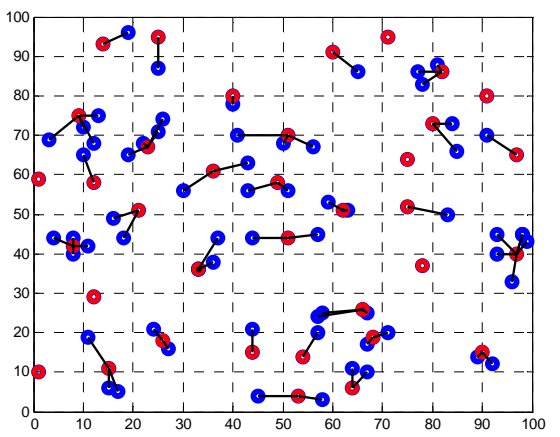

Fig. 5. Transmission range is set to $10 \mathrm{~m}$ with 100 nodes

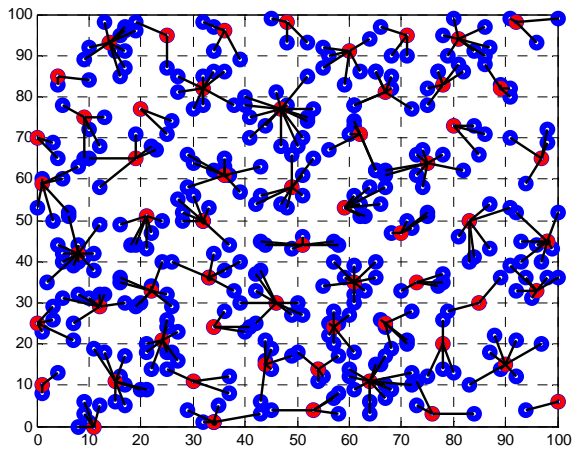

Fig. 6. Transmission range is set to $10 \mathrm{~m}$ with 400 nodes

It can be seen in Fig. 5 that when transmission range is $10 \mathrm{~m}$ with 100 nodes, there are more number of single-node cluster heads. In contrast, in a dense network, the number of single-node cluster head decreases as shown in Fig. 6. It can be observed that when the transmission range is short, the number of cluster heads elected is high and causes more relaying between cluster heads to forward data to BS. We observed for a sparse network, a connected network can be obtained by increasing the transmission range of cluster head whereas for dense network, connected network can be obtained through gateways nodes.

\section{Minimizing the Dominating set}

It can be observed from Fig.7 and Fig. 8 that when transmission range is increased to $30 \mathrm{~m}$, small number of cluster heads elected compared to Fig.5 and Fig.6 where more cluster heads are elected. The transmission range determines the size of cluster and if small cluster is created the energy consumption for member nodes to transmit to their cluster heads will be minimized.

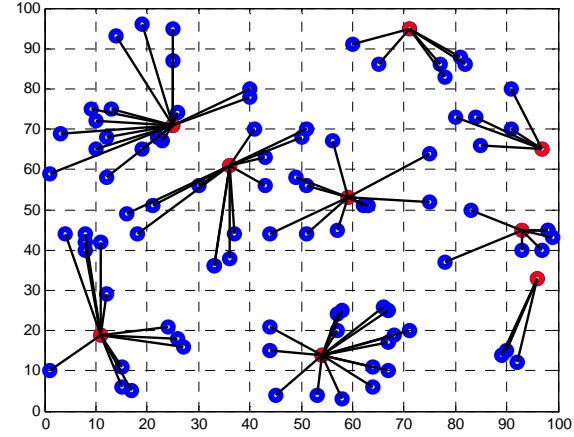

Fig. 7. Transmission range is set to $30 \mathrm{~m}$ with 100 nodes

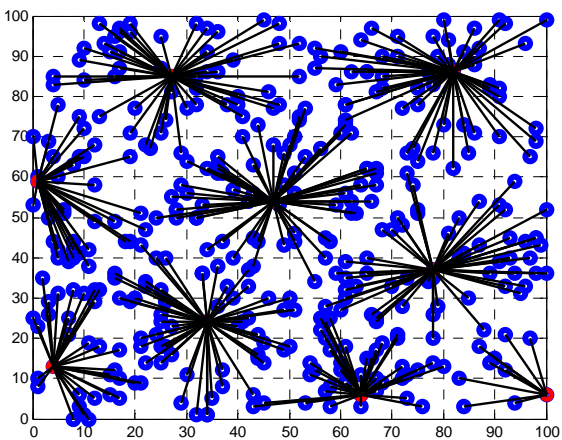

Fig. 8. Transmission range is set to $30 \mathrm{~m}$ with 400 nodes.

\section{CONCLUSIONS}

The experimental results demonstrate new hybrid approach of constructing dominating set by combining energy-efficient technique of clustering and power control to obtain a dominating set in Wireless Sensor Networks. The proposed hybrid protocol constructs dynamic dominating set based on energy and coverage metrics with low computational complexity and significant reduction in amount of message overhead complexity of $\mathrm{O}(n)$ shown in the simulation results. Through power level, the ability to control the number of dominating sets and cluster heads can be achieved and therefore, one-hop dominating set can be obtained. It is shown that the dominating set is dynamically constructed at every round to achieve balanced load distribution. This approach can be extended for constructing energy-efficient connected dominating set in Wireless Sensor Networks.

\section{REFERENCES}

[1] P. M. Wightman and M. A. Labrador, "A Topology Construction Algorithm for Wireless Sensor Networks," IEEE Global Telecommunications Conference, 2008, pp. 1-6.

[2] A. Förster, A. Förster, and A. L. Murphy, "Optimal Cluster Sizes for Wireless Sensor Networks," An Experimental Analysis: Ad Hoc Nets, 2009, pp. 49-63.

[3] B. Yin, "Adaptive Clustering and Transmission range adjustment for Topology Control in Wireless Sensor Networks," Ph.D. thesis, University of Missouri-Columbia, 2006.

[4] J. Li, L. L. Andrew, C. H. Foh, M. Zukerman, and H. H. Chen, "Coverage and Placement in Wireless Sensor Networks," Sensors, 2009, vol. 9, no. 10, pp. 7664-7693.

[5] R. Wattenhofer, "Algorithms for ad hoc and sensor networks," Computer Communications, 2005, vol. 28, no. 13, pp. 1498-1504.

[6] P. M. Wightman and M. A. Labrador, Topology Control in Wireless Sensor Networks - with a companion simulation tool for teaching and research, Springer. 
[7] F. Kuhn and R. Wattenhofer, "Constant-time distributed dominating set approximation," in Proc. of Distributed Computing, 2005, vol. 17, no. 4, pp. 303-310.

[8] A. Förster, Teaching Networks How to Learn, Reinforcement Learning for Data Dissemination in Wireless Sensor Networks, Ph.D. Dissertation, University of Lugano.

[9] B. Chen, K. Jamieson, H. Balakrishnan, and R. Morris, "An Energy-Efficient Coordination Algorithm for Topology Maintenance in Ad Hoc Wireless Networks," Journal Wireless Networks, vol. 8, no. 5, 2002.

[10] W. R. Heinzelman, A. P. Chandrakasan, and H. Balakrisnan, “An application-specific protocol architecture for wireless micro sensor networks," IEEE Transactions on Wireless Communications, 2002, vol. 1, no. 4, pp. 660-670.

[11] M. Ye, C. F. Li, G. H. Chen, and J. Wu, "an energy efficient clustering scheme in wireless sensor networks," IEEE International Performance Computing and Communications Conference, 2005, pp. 535-540.

[12] A. A. Abbasi and M. F. Younis, "A survey on clustering algorithms for Wireless Sensor Networks," Computer Communications, 2007, vol. 30 pp. 2826-2841.

[13] N. Aslam, W. Robertson, W. Phillips, and S. Sivakumar, "A Multi-Criterion optimization technique for energy efficient cluster formation in Wireless Sensor Networks," Information Fusion Journal, manuscript ID: IF08A04-FGC.

[14] O. Younis and S. Fahmy, "A Hybrid, energy-efficient distributed clustering approach for Ad Hoc Sensor Networks," IEEE Transactions on Mobile Computing, 2004, vol. 3, no. 4, pp. 660-669.
[15] S. George, I. Matta, and A. Bestavros, "A Stable Election Protocol for clustered heterogeneous Wireless Sensor Networks," in Proceedings of Second International Workshop on Sensor and Actuator Network Protocol and Applications, 2004

[16] L. Bao and J. J. G. L. Aceves, "Topology management in ad hoc networks," in Proceedings of the 4th ACM International Symposium on Mobile Ad Hoc Networking and Computing, 2003, pp. 129-140.

[17] L. Jia, R. Rajaraman, and T. Suel, "An efficient distributed algorithm for constructing small dominating sets," Distributed Computing, 2002, vol. 15 , no. 4 , pp. 193-205

[18] M. R Gary and D. S Johnson, Computers and Intractability, a Guide to the theory of NP-completeness, W. H. Freeman and Company, 1979

[19] R. Wattenhofer, "Algorithms for ad hoc and sensor networks," Computer Communications, 2005, pp. 1498-1504.

[20] H. K. Qureshi, S. Rizvi, M. Saleem, S. A. Khayam, V. Rakocevic, and M. Rajarajan, "A Reliable and Energy Efficient Topology Control Protocol for Wireless Sensor Networks," Computer Communications, 2011, vol. 34, no. 10, pp. 1235-1242.

[21] M. Kubisch, H. Karl, A. Wolisz, L. Zhong, and J. Rabaey, "Distributed algorithms for transmission power control in wireless sensor networks," in Proc. of the IEEE Wireless Communications and Networking Conference, 2003, vol. 1, pp. 558-563.

[22] A. Hać, Wireless Sensor Networks Designs, John Wiley \& Sons Ltd, 2003

[23] C. M. Cordeiro and D. P. Agrawal, Ad Hoc and Sensor Networks, Theory and Applications, World Scientific, 2006 